\title{
E04 SYMMETRIC POSITIVE DEFINITE GENERAL TENSOR DISCRETIZATION OPERATORS ON UNSTRUCTURED AND FLOW BASED GRIDS
}

\author{
Michael G Edwards \\ Civil and Computational Engineering Centre \\ University of Wales Swansea \\ Singleton Park \\ Swansea UK
}

\section{Summary}

The derivation of algebraic flux continuity conditions for full tensor discretization operators has lead to efficient and robust locally conservative flux continuous finite volume methods for determining the discrete velocity field in subsurface reservoirs e.g [1-8].

New locally conservative flux continuous formulations are presented for unstructured grids comprised of quadrilateral and or triangular grid cells. The new schemes guarantee that a symmetric positive definite discretization matrix is obtained for an arbitrary polygonal control-volume distributed formalism. The relationships between the new formulation and earlier flux continuous schemes are given. M-matrix conditions of the schemes are outlined and the relationship with more traditional CVFE based methods is also presented [9]. A flow based grid generation process is also briefly described which involves concentrating grid nodes in high flow paths that are detected via a single phase flow response $[6,10]$. Method performance is demonstrated on unstructured flow based grids for two phase flow problems.

\section{Flow Equations}

While the methods presented here are for both single phase and multiphase flow problems, specific discretization issues are considered that arise with the finite volume approximation of the pressure equation on general unstructured grids. Further details of the discrete coupled formulation can be found in [6]. We shall begin with the integral form of the pressure equation written as

$$
\int_{\Omega} \nabla \cdot \mathbf{V} d \tau=\int_{\partial \Omega}(-\mathbf{K} \nabla \phi) \cdot \hat{\mathbf{n}} d s=M
$$

where $\mathbf{V}$ is the Darcy velocity, $\mathbf{K}$ the permeability tensor, $\phi$ is the pressure and $\Omega$ is a representative control-volume. Neumann zero flux conditions apply on solid walls. Inflow-outflow conditions apply at wells where fluxes/pressures are prescribed. Transforming to curvilinear coordinates $\left(\xi_{1}, \xi_{2}\right)$, and using the chain rule the spatial derivatives take the form $\phi_{x}=J(\phi, y) / J(x, y), \quad \phi_{y}=J(x, \phi) / J(x, y)$

where the Jacobian determinant $J$ is given by $J(x, y)=x \xi_{1} y \xi_{2}-x \xi_{2} y_{\xi_{1}}$. The flux in Eq. 1 is now resolved along the outward normals to constant $\xi_{1}$ and $\xi_{2}$ respectively, where normal increments are

$$
\mathbf{n}_{1}=\hat{\mathbf{n}}_{1} d s_{1}=\left(y \xi_{2},-x_{\xi_{2}}\right) d \xi_{2}, \mathbf{n}_{2}=\hat{\mathbf{n}}_{2} d s_{2}=\left(-y \xi_{1}, x \xi_{1}\right) d \xi_{1} \text {. }
$$

This gives rise to the general tensor form of the pressure equation

$$
\oint_{\partial \Omega_{p}} \mathbf{V} \bullet \hat{\mathbf{n}} d s=\sum_{i=1}^{2} \Delta_{\xi_{i}} \mathrm{~F}_{i}=M
$$

where the outward normal flux is given by 
$\mathrm{F}_{i}=-\int \sum_{j=1}^{2} T_{i j} \phi_{\xi} d \Gamma_{i}$

where $\xi_{i}$ are curvilinear parametric coordinates, $d \Gamma_{i}$ is the parametric coordinate surface increment and $\phi_{\xi_{j}}$ is the derivative of $\phi$ with respect to $\xi_{j}$. The $T_{i j}$ are components of the general tensor

$\mathbf{T}=J \mathbf{J}^{-1} \mathbf{K} \mathbf{J}^{-T}$

which is in turn a function of the Cartesian permeability tensor $\mathbf{K}$ and geometry, where $\mathbf{J}=\partial \mathbf{x} / \partial \xi$ is the Jacobian of the curvilinear coordinate transformation, further details are given in [1].

\section{Finite Volume Schemes}

In this paper control-volume distributed flux-continuous (CVD) schemes are considered for general unstructured grids in 2-D, where flow variables are assigned to the grid vertices and rock properties are assigned to control-volumes, and e.g. permeability is piece-wise constant over each polygonal controlvolume. For pure triangulations the grid definition follows the point-distributed formalism [5]. In the case of structured (logically Cartesian) grids, the methods presented also apply to cell (block) centered formulations [1-3]. The primal grid considered here can be a hybrid composed of triangles and/or quadrilateral cells of arbitrary structure. A polygonal control-volume is built around each grid vertex by joining cell centers with cell edge mid-points for all cells that share a common grid vertex, figures $1 \mathrm{a}, 1 \mathrm{~b}$ and is therefore comprised of a set of sub-quadrilateral cells. Consequently each primal grid cell is composed of three (for a triangle) or four (for a quadrilateral) sub-cells, Fig. 1.

Space restrictions prevent a complete overview of the multiphase coupled scheme construction, instead a summary is given in this section for single phase flow. The discrete pressure equation is derived in two steps: (a) A unique discrete flux is defined along the normal to each sub-cell control-volume face, Fig 1, (see below). This gives rise to four fluxes per quadrilateral $F_{N_{j_{1}}}, F_{S_{j_{1}}}, F_{E_{j_{1}}}, F_{W}$ and three per triangle $F_{N_{j_{2}}}, F_{S_{j_{2}}}, F_{E_{j_{2}}}$ (b) After application of the Gauss divergence theorem over each controlvolume, the discrete integral of divergence is defined by the sum of net edge based fluxes

$\sum_{k=1}^{N e v} F_{e(k, i)}(\phi)=M$

where summation is over all edges $e(k, i)$ (local edge index $k$ ) passing through the ith grid vertex. This flux is comprised of the sum of adjacent sub-cell fluxes that belong to the primal grid cells with common edge $e(k, i)$. This is illustrated with reference to vertex $i$ and edge $k$ of Fig. $1 \mathrm{~b}$ and the local fluxes of Fig. 1 , in this case the net edge based flux is given by $F_{e(1, i)}=F_{E_{j_{1}}}+F_{N_{j_{2}}}$. Further details are given in [6]. Sub-cell flux approximations are briefly described below.

\section{Control-Volume Flux and Continuity in Two Dimensions}

Consistent flux approximations that respect continuity in pressure and flux across the control-volume interfaces within each primal grid cell are presented in [4-8]. These schemes involve the introduction of auxiliary continuous interface pressures, one per control-volume sub-face as indicated in Fig. 1a. Subcell triangular basis functions are then formed by joining the cell vertices, e.g. a triangle cell with locally numbered vertex pressures $\boldsymbol{\Phi}_{\mathbf{V}}=\left(\phi_{1}, \phi_{2}, \phi_{3}\right)$, to the positions of the adjacent interface pressures, again for a triangle interface pressures $\boldsymbol{\Phi}_{\mathbf{f}}=\left(\phi_{N}, \phi_{S}, \phi_{E}\right)$. Two sub-cell normal fluxes are then defined per sub-triangle and flux continuity is imposed by equating fluxes on the left and right hand sides of each interface. The continuity conditions are summarized below for a triangular cell, further details for quadrilaterals and triangles are given in [6]. Using the general tensor form of the flux results in three flux continuity conditions 


$$
\begin{aligned}
& F_{S}=-\left.\frac{1}{2}\left(T_{11} \phi_{\xi}+T_{12} \phi_{\eta}\right)\right|_{S} ^{1}=-\left.\frac{1}{2}\left(T_{11} \phi_{\xi}+T_{12} \phi_{\eta}\right)\right|_{S} ^{2} \\
& F_{E}=-\left.\frac{1}{2}\left(T_{12} \phi_{\xi}+T_{22} \phi_{\eta}\right)\right|_{E} ^{2}=-\left.\frac{1}{2}\left(T_{12} \phi_{\xi}+T_{22} \phi_{\eta}\right)\right|_{E} ^{3} \\
& F_{N}=-\left.\frac{1}{2}\left(T_{12} \phi_{\xi}+T_{22} \phi_{\eta}\right)\right|_{N} ^{1}=-\left.\frac{1}{2}\left(T_{11} \phi_{\xi}+T_{12} \phi_{\eta}\right)\right|_{N} ^{3}
\end{aligned}
$$

In this case approximations of the derivatives $\phi_{\xi}, \phi_{\eta}$ are linear functions of $\boldsymbol{\Phi}_{\mathbf{V}}$ and $\boldsymbol{\Phi}_{\mathbf{f}}$, and $\left.\Gamma\right|_{\sigma} ^{j}$ denotes interface flux $\Gamma$ at location $\sigma$ defined with respect to sub-cell $j$. The three interface pressures are eliminated in terms of the three vertex pressures using Eq. 7 to yield generalized discrete fluxes that embody the full tensor generalization of the harmonic mean.

\section{Loss of Symmetry and Stability of Standard Flux}

Imposition of the standard discrete continuity conditions in physical space leads to a discretization matrix that is non-symmetric in the general case for both quadrilateral and triangular grid cells. In the analysis that follows focus is on triangle sub-cell 1 Fig. 1 and it is understood that other sub-cell contributions can be treated by an analogous procedure and lead to identical conclusions. In this case the fluxes $F_{S}$ and $F_{N}$ of Eq. 7 are evaluated with respect to sub-cell 1 at locations $\mathrm{N}$ and $\mathrm{S}$ and so in general $T_{12 N}^{1} \neq T_{12 S}^{1}$, leading to a loss of symmetry since the local discrete tensors are defined at two distinct continuity points of each control-volume sub-cell. The actual tensor coefficients of Eq. 7 can be identified in a frame of reference aligned with respect to the primal grid triangle. First fluxes of sub-cell 1 are defined by resolving the piecewise constant sub-triangle velocity vector along the two control-volume normals of Fig 1. With the center of gravity of the primal triangle defined by $\mathbf{r}_{M}=\left(\mathbf{r}_{1}+\mathbf{r}_{2}+\mathbf{r}_{3}\right) / 3$ and edge midpoints by $\mathbf{r}_{S}=\left(\mathbf{r}_{1}+\mathbf{r}_{2}\right) / 2, \mathbf{r}_{N}=\left(\mathbf{r}_{1}+\mathbf{r}_{3}\right) / 2$, then the control-volume face-tangent vectors are $\mathbf{r}_{M}-\mathbf{r}_{S}$ and $\mathbf{r}_{N}-\mathbf{r}_{M}$ respectively. Denoting e.g. $x_{21}=x_{2}-x_{1}$, the respective two normal vectors are

$$
\begin{aligned}
& \left(y_{M}-y_{S},-\left(x_{M}-x_{S}\right)\right)=\left(2\left(y_{31},-x_{31}\right)+\left(-y_{21}, x_{21}\right)\right) / 6 \\
& \left(y_{N}-y_{M},-\left(x_{N}-x_{M}\right)\right)=\left(\left(y_{31},-x_{31}\right)+2\left(-y_{21}, x_{21}\right)\right) / 6
\end{aligned}
$$

When using piecewise linear basis functions over each triangle, vector derivatives $\mathbf{r}_{\xi}=\left(x_{\xi}, y_{\xi}\right)$, $\mathbf{r}_{\eta}=\left(x_{\eta}, y_{\eta}\right)$ are approximated by the differences $\left(\mathbf{r}_{2}-\mathbf{r}_{1}\right)=\left(x_{21}, y_{21}\right)$ and $\left(\mathbf{r}_{3}-\mathbf{r}_{1}\right)=\left(x_{31}, y_{31}\right)$ respectively. So that by the definitions in Eq. 2, it follows that the normal vectors of Eq. 8 can be identified as consistent discrete approximations of

$\left(2 \mathbf{n}_{1}+\mathbf{n}_{2}\right) / 6$ and $\left(\mathbf{n}_{1}+2 \mathbf{n}_{2}\right) / 6$

respectively. Therefore the two physical flux components corresponding to the $(\xi, \eta)$ directions can be expressed with respect to the discrete geometry tensor of the primal grid triangle (c.f. Eq. 3) where

$\mathbf{F}=-\frac{2}{3}\left(\begin{array}{cc}T_{11}^{1}+\frac{1}{2} T_{12}^{1} & T_{12}^{1}+\frac{1}{2} T_{22}^{1} \\ T_{12}^{1}+\frac{1}{2} T_{22}^{1} & T_{22}^{1}+\frac{1}{2} T_{12}^{1}\end{array}\right)\left(\begin{array}{l}\phi_{S}-\phi_{1} \\ \phi_{N}-\phi_{1}\end{array}\right)$

yielding a non-symmetric flux matrix. For special cases a symmetric scheme can be obtained by moving the triangle mid-point [8]. Note by Eq. 10 that the leading (diagonal) two-point flux coefficients are conditionally positive, implying that it is possible to obtain countercurrent flow. For example, if the 
interface pressures at $S$ and $N$ have a lower pressure than node 1 and the tensor coefficients satisfy $T_{12}<0, T_{11}<\left|T_{12}\right| / 2$. This latter observation has four important consequences:

1. If the above conditions hold over a triangle the flux in the CVFE formulation can be anti-diffusive, by Eq. 11 below.

2. If the conditions hold over a triangle sub-cell there is potential for anti-diffusion to be introduced into the flux continuity formulation.

3. In addition to anti-diffusion, the general simulator formulation may be accompanied by incorrect upwinding being applied for multiphase flow with potential for further instability.

4. Flux splitting of [7] can become unstable as this relies upon the leading two-point flux as the driving mechanism of the scheme.

\section{Relationship with CVFE}

If permeability is constant over each primal grid triangle then the flux of Eq. 10 reduces to

$\mathbf{F}=-\frac{1}{3}\left(\begin{array}{ll}T_{11}+\frac{1}{2} T_{12} & T_{12}+\frac{1}{2} T_{22} \\ T_{12}+\frac{1}{2} T_{11} & T_{22}+\frac{1}{2} T_{12}\end{array}\right)\left(\begin{array}{l}\phi_{2}-\phi_{1} \\ \phi_{3}-\phi_{1}\end{array}\right)$

and for single-phase flow the above scheme produces an identical discretization matrix to that of the CVFE scheme, since the sub-triangle tensor is identical to the triangle tensor for constant permeability (thus the superfix is removed) and summing fluxes of Eq. 11 over the two sub-cell control-volume faces

$$
F_{1}+F_{2}=-\frac{1}{2}\left(\left(T_{11}+T_{12}\right)\left(\phi_{2}-\phi_{1}\right)+\left(T_{12}+T_{22}\right)\left(\phi_{3}-\phi_{1}\right)\right)
$$

The flux of Eq. 12 is also equal to the total general tensor CVFE flux that passes through line $N S$. While this is independent of control-volume geometry inside the triangle, it is dependent on the primal triangle edge geometry tensor. For a diagonal permeability tensor the flux of Eq. 12 corresponds to the CVFE flux of [9]. Corollary: A symmetric flux matrix is not a necessary condition for a symmetric discretization matrix, since CVFE is symmetric. However the continuous CVD scheme is not necessarily symmetric.

M-matrices and Diagonal Dominance: Cell-wise diagonal dominance analysis is performed by focusing upon local node 1 of the triangle and assembling the cell-wise matrix contributions to the diagonal (at node1) and off-diagonal terms (nodes 2 and 3). For the case of a general tensor it follows that the above scheme (and therefore the CVFE scheme) has an M-matrix (subject to a Dirichlet boundary condition) with weak diagonal dominance with positive diagonal and negative off-diagonal coefficients if

$$
\left|T_{12}\right|<\min \left(T_{11}, T_{22}\right)
$$

and that the matrix is irreducible. Eq. 13 is the general condition for ensuring that the pressure field is monotonic, and when expressed in this form the requirement for positive coefficients of triangle edge pressure differences leads at once to the conditions for both diagonal dominance and positive transmissibility. In particular if Eq. 1 is reduced to the Laplacian, diagonal dominance holds with Eq 13 satisfied, if the maximum angle of each triangle satisfies $\theta \leq \pi / 2$, and in this case diagonal dominance holds if the domain is convex and the triangulation is Delaunay [9].

Note that in general, if Eq. 13 is satisfied, then positive leading coefficients are certainly guaranteed within the local flux matrices defined in Eq's. 10 and 11. Conversely this implies that loss of diagonal dominance may have a direct influence on stability within all of the above schemes. E.g. since upwinding is performed as a function of the net sub-cell flux direction. 


\section{Symmetric Positive Definite Flux-Continuous Formulations}

A symmetric positive definite formulation is obtained for the flux continuous control-volume distributed formulation if pairs of sub-cell fluxes are defined with respect to the same piece-wise constant symmetric general tensor per sub-cell. Thus symmetry of the flux matrix is a sufficient condition for a symmetric discretization matrix. A related approach has been used previously for quadrilateral grids [1,2], when formulated in transform space these formulations have piece-wise constant general tensors defined with respect to each control-volume. It is proven in [6] that when using a piece-wise constant general tensor per sub-cell the flux continuous schemes are symmetric positive definite for any grid comprised of quadrilateral and/or triangular cells. One of the key issues in this new formulation is the identification of the appropriate general tensor for a sub-cell.

In this paper three possibilities are considered:

1.0 Local Mapping: The first definition is a natural generalisation of that described in [1], which corresponds to defining a local mean general tensor corresponding to the local sub-quadrilateral of each triangle. In this approach the sub-quadrilateral cells are in effect mapped to unit cells, Fig $2 \mathrm{a}$.

2.0 Control-volume Face Tensor: In the second definition, the piece-wise constant tensor is defined with respect to the sub-cell control-volume face geometry, Fig $2 \mathrm{~b}$. The local general tensor is built in a coordinate system directly aligned with the two control-volume faces, so that the surface normal vectors are naturally incorporated within the formulation. This formulation is most closely associated with the physical control-volume faces and is robust provided that no pair of control-volume sub-cell faces are parallel.

3.0 Cell-Edge Tensor Flux Balance: In the third definition, the local general tensor is built in a coordinate system aligned with the primal triangle edges and uses the natural geometry tensor of the triangle. Referring to sub-cell 1 , the two control-volume face fluxes of are defined by

$$
\mathbf{F}=-\left(\begin{array}{ll}
T_{11}^{1} & T_{12}^{1} \\
T_{12}^{1} & T_{22}^{1}
\end{array}\right)\left(\begin{array}{l}
\phi_{S}-\phi_{1} \\
\phi_{N}-\phi_{1}
\end{array}\right)
$$

which are also equal to the fluxes flowing into the sub-cell through the triangle half-edges. Thus in this case the continuity conditions lead to a direct balance of triangle edge based fluxes, Fig $2 \mathrm{c}$, and the subcell flux integrals are independent of control-volume geometry inside the triangle, which may have a beneficial effect, see results. (If the permeability tensor is piece-wise constant over each primal triangle and if the edge mid-point pressures are equal to the average of the respective two edge vertex pressures then the sum of fluxes $F_{1}+F_{2}$ is equal to the Galerkin CVFE flux.) In summary each of the three above CVD schemes possesses a symmetric sub-cell flux matrix and leads to a symmetric positive definite discretization with unconditionally positive leading two-point flux coefficients, ensuring counter-current flow cannot occur, i.e. a consistent flow direction is defined by the sub-cell fluxes.

Quadrilaterals, Triangles and Consistency: Details of the flux continuous formulations for quadrilateral grids are given in $[1,6]$. Any one of the three above sub-cell tensor approximations can be employed for quadrilateral grid cells and can be expected to lead to a consistent scheme. Convergence studies of a related scheme are given in [1]. In this paper the latter two are compared for both hybrid quadrilateraltriangle grids and pure triangulations. For triangles, the first two of the three above schemes introduce an additional approximation with respect to local geometry. However, geometry is exact in the third (edgebased) scheme.

\section{Flow Based Grids}

The gridding techniques presented here are primarily focused on unstructured static flow based grid generation [6]. In the case of a heterogeneous reservoir detection of connectivity is particularly important. An a priori estimate of the flow field is used to detect important areas such as high velocity regions of the reservoir. Following $[10,6]$, a single-phase steady-state flow response is used, subject to fixed pressure boundary conditions specified on the left and right hand walls respectively and zero flow on the top and

$8^{\text {th }}$ European Conference on the Mathematics of Oil Recovery - Freiberg, Germany, 3 - 6 September 2002 
bottom walls. We shall refer to these boundary conditions as the mean flow conditions. However, the flow response is clearly boundary condition dependent and an appropriate selection requires further research. Since streamline density or flux is automatically concentrated in high flow regions the local steady state flux is used to develop a refinement indicator for a static grid.

The underlying unstructured grid generator described here is based on a quad-tree algorithm, together with triangulation of the interfaces. The nested grid is converted into either an unstructured grid comprised of quadrilateral and triangular cells (hybrid grid), or an entirely unstructured triangulation is defined. Local grid refinement is performed according to the streamline density distribution. Laplacian based smoothing is applied to ensure an improved grade of nodal distribution [6]. In addition equidistribution is also developed which involves iteratively solving

$\nabla \bullet \mathbf{W} \nabla \mathbf{r}=0$.

rather than using the Laplacian, where $\mathbf{W}$ is a function of flux and permeability and $\mathbf{r}=(x, y)$, c.f. Fig 5 .

The final grid is defined by the dual where permeability is control-volume distributed.

Upscaling for Point Distributed Schemes: Distributing permeability to control-volumes involves defining an upscaling region or bounding box that spans the minimum to maximum coordinates of each controlvolume polygon. Standard upscaling algorithms can then be applied to the bounding box, Fig $2 \mathrm{~d}$. This approach was motivated by the boarder region approach described in [11] and may be viewed as one possible generalization of the method for general grids.

\section{Results}

The performance of the finite volume schemes and flow based grids are examined for two phase flow through a heterogeneous domain Fig 3a, with a full tensor permeability field with principal axes inclined at 45 degrees. An injecting well and producing well are placed as indicated with symbols $I$ and $P$ respectively in Fig 3a. The solution is computed using the standard flux continuous scheme c.f. Eq. 10. The uniform grid water saturation contours are shown after breakthrough (100 days) in Fig 3c.

The flow based grid is generated from a single phase flow response, computed using the mean flow conditions defined above. In this case flow is dominated by the strong cross terms in the permeability tensor, which drives the flow diagonally across the domain. Local refinement is performed according to streamline flux and the resulting flow based dual grid (600 vertices) is shown in Fig 3a. The local grid density clearly reflects the dominant flux field. However, unlike a truly dynamic grid such global resolution can never be optimal, but for a given reservoir, it may provide a good static grid that is suitable for a number of different boundary conditions. The permeability field is upscaled to the control-volume dual grid and shown in Fig 3a. The dual grid (thick lines) and the primal triangular grid (thin lines) are also shown in Fig $3 a$.

The standard flux continuous CVD scheme is compared with the second symmetric positive definite scheme. Flow solutions computed by both CVD schemes on the flow based grid are compared with that of the uniform grid solution with contours in Fig's 3c, 3d, 3e and fractional flow in Fig 3b. While the static grid captures the flow in an average sense, the limitation of a static grid is also evident in the contour plot. As is to be expected, whenever the front traverses the finer grid interfaces the local resolution deteriorates. However, the flow based grid fractional flow results (oil) are close to that of the uniform grid, predicting very good agreement in break-through time while uniform grid simulation time is reduced by $40 \%$. The results show that the new scheme is in good agreement with the standard method.

In the second case a similar comparison of schemes is performed for a heterogeneous channeled domain (from [12]). Again injecting and producing wells are placed as indicated with symbols $I$ and $P$ respectively in Fig 4a. The flow based grid (dual in Fig 4a with 669 vertices) is generated from a single phase flow response (with mean flow boundary conditions differing to the problem in hand). Permeability is upscaled to the control-volume dual mesh. A comparison is presented between the uniform grid 
standard CVD scheme Fig 4c, standard CVD on the flow based grid Fig 4d and the new CVD scheme 3.0 on the flow based grid Fig 4e. A 35\% saving is made using the flow based grid while good agreement in fractional flow (oil) is obtained. The new CVD scheme 3.0 produces further improvement in fractional flow (Fig4b) compared to the standard scheme, which is also reflected in the contours, Fig 4e versus $4 \mathrm{~d}$.

Finally, a relatively course flow-based hybrid grid (211 nodes Fig 5a) is further enhanced via spatial equidistribution using a function of streamline density within the divergence operator c.f. Eq. 15. A comparison between the course adapted flow based grid and uniform grid result are presented for the same channeled case, showing a sharp improvement in breakthrough Fig $5 \mathrm{~b}$.

\section{Conclusions}

New locally conservative flux-continuous, control-volume distributed, discretization schemes are presented for general unstructured grids. The new formulations ensure that general symmetric positive definite discretization matrices are obtained for any quadrilateral, triangular or unstructured hybrid grid.

Examination of the generalized leading diagonal coefficients shows that the standard CVD scheme can give rise to negative diagonal coefficients. In particular M-matrix conditions point to the limitation of traditional CVFE based approaches versus the new formulations. The new scheme guarantees that the discrete leading diagonal tensor coefficients are unconditionally positive.

A novel flow based unstructured mesh generator is presented for heterogeneous reservoir domains. A significant reduction in computation time can be achieved with the static flow-based gridding strategy while maintaining close agreement with respect to fine grid global flow parameters. A comparison between the standard and new CVD schemes is performed on the flow based grids and demonstrates the consistency and potential benefits of the new schemes.

\section{References}

1 Edwards M.G. and Rogers C.F "Finite Volume Discretization with Imposed Flux Continuity for the General Tensor Pressure Equation " Computational Geosciences vol 2 1998, pp 259-290.

2 Aavatsmark I., Barkve T., O. Boe and T Mannseth, Discretization on Non-Orthogonal, Quadrilateral Grids for Inhomogeneous Anisotropic Media. J. Comput. Phys. 127 1996, 2-14

3 Lee S. H. Durlofsky L. J. Lough M. F. and Chen W. "Finite Difference Simulation of Geologically Complex Reservoirs With Tensor Permeabilities. SPE 38002 1997, SPE Reservoir Simulation Symposium, Dallas TX

4 Avatsmark A., T. Barkve, O. Boe and T. Mannseth, "Discretization on Unstructured Grids for Inhomogeneous, Anisotropic Media" Part 1 Methods SIAMJ Sci. Comput. 19, (1998), 1700-1716

5 Verma S. and Aziz K. "A Control Volume Scheme for Flexible Grids in Reservoir Simulation" SPE 37999 14th SPE Reservoir Simulation Symposium, Dallas, Texas, USA, pp 215, 227, 8-11 June 1997

6 Edwards M G "Unstructured, Control-Volume Distributed, Full-Tensor Finite Volume Schemes With Flow Based Grids" to appear: Computational Geosciences

7 Edwards M G "M-matrix Flux Splitting for General Full Tensor Discretization Operators on Structured and Unstructured Grids" J. Comput. Phys 160, pp 1-28, 2000

8 Eigestad G. T, I. Aavatsmark, M. Espedal "Symmetry and M-matrix Issues for the O-method on an Unstructured Grid" to appear: Computational Geosciences

9 Forsyth $\mathrm{P}$ “A Control-Volume Finite Element Method for Local Mesh Refinement in Thermal Reservoir Simulation" SPERE Nov 1990 p 561 
10 Castellini A, Edwards M G and Durlofsky L J "Flow Based Modules for Grid Generation in Two and Three Dimensions" proc: 7th European Conference on the Mathematics of Oil Recovery, Italy Sept $5-8^{\text {th }} 2000$

11 Wen X.H., L.J. Durlofsky and M.G. Edwards, "Upscaling of Channel Systems in Two Dimensions Using Flow Based Grids" to appear: Transport in Porous Media

12 Mao S and Journal A. G. "Generation of a Reference Petrophysical Data Set: The Stanford V Reservoir. Stanford Center for Reservoir Forecasting Report.

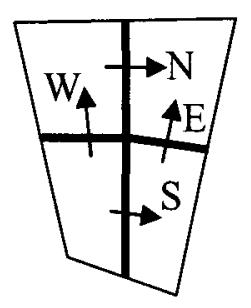

Primal Grid Dual Grid

Fig 1a: Sub-cell Fluxes N,S,E,W

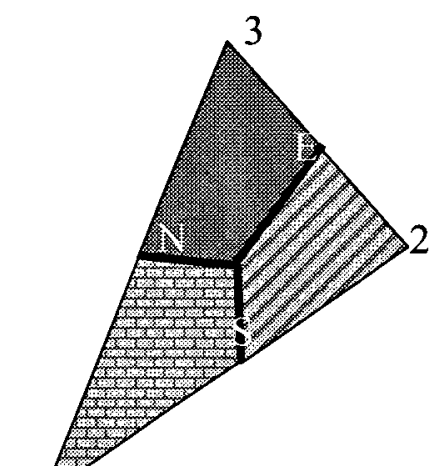

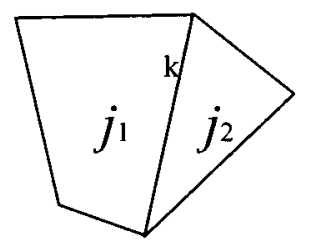

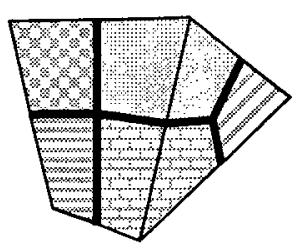

Fig 1b: Two Cell Segment of Primal Grid Control-volume permeability is shaded

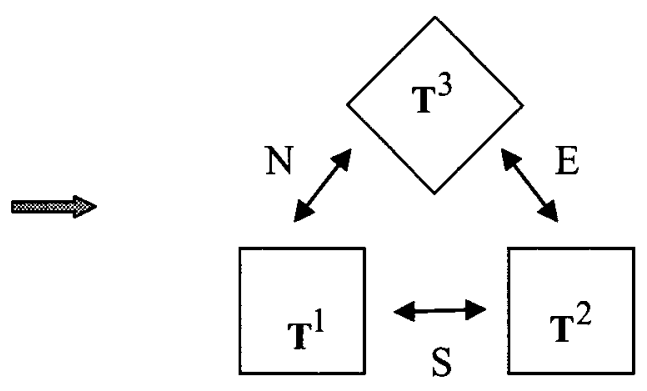

Fig 2a: Mean sub-cell tensors and sub-quadrilateral mapping/connectivity 


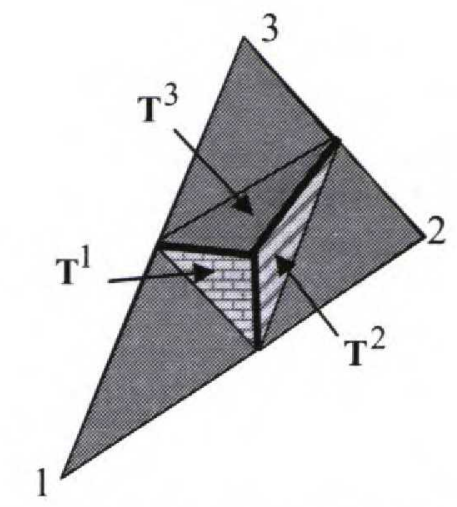

Fig 2b: Sub-cell tensors using controlvolume face triangles

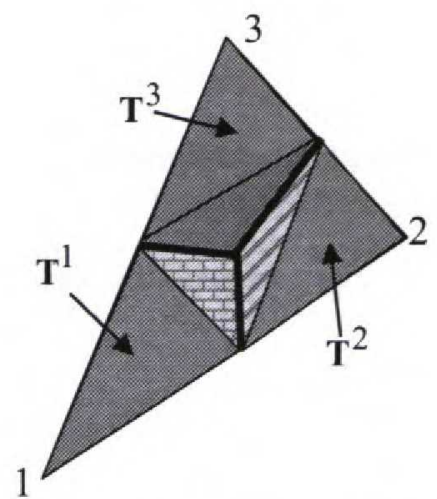

Fig 2c: Sub-cell tensors using sub-triangles

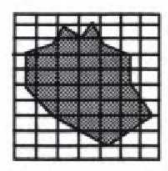

Fig 2d: Control-volume (shaded) and Fine-scale Bounding Box for Upscaling

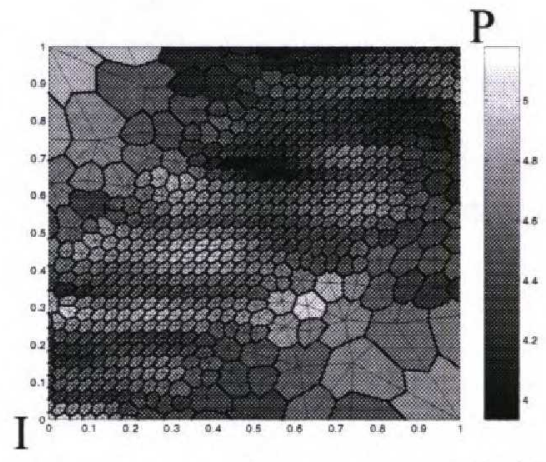

3a: Flow Based Triangle Dual Grid

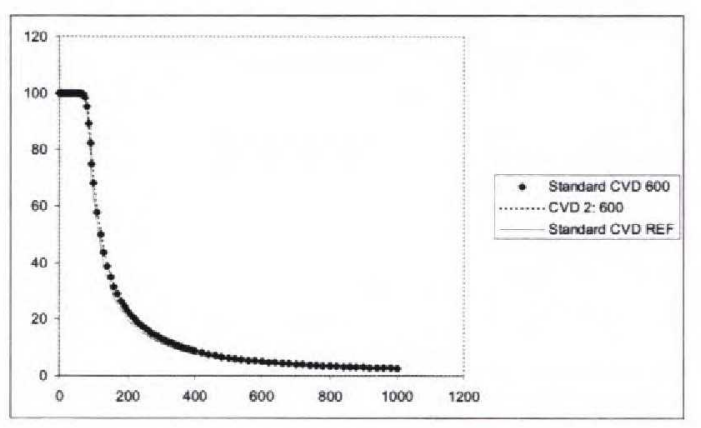

3b: Fractional Flow Comparison

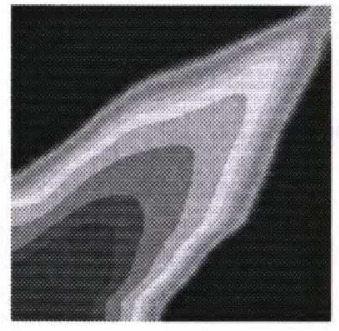

3c: Uniform Triangle Dual Standard CVD Scheme

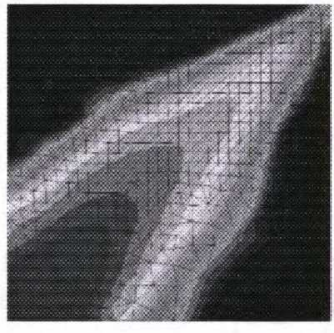

3d: Flow Based DualStandard CVD Scheme

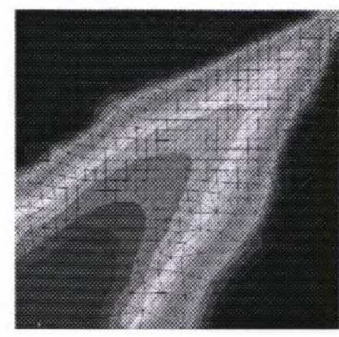

3e: Flow Based Dual CVD Scheme 2 


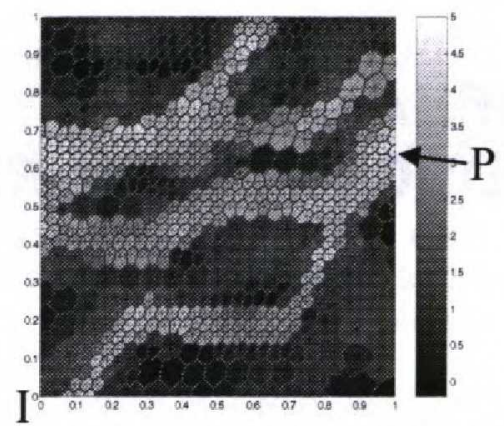

4a: Flow Based Triangle Dual Grid

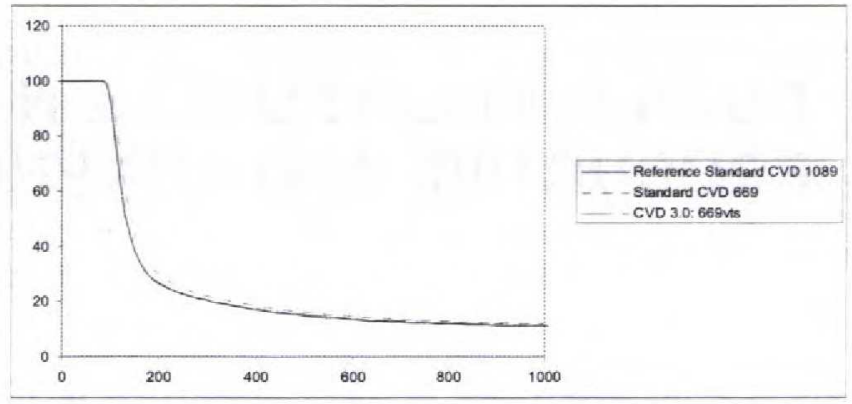

4b: Fractional Flow Oil oilComparison

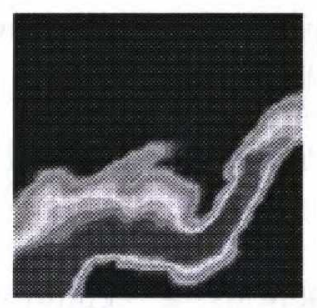

4c: Uniform Triangle Dual Standard CVD Scheme

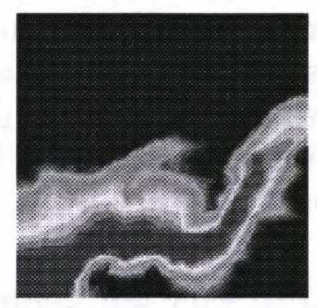

4d: Flow Based DualStandard CVD Scheme

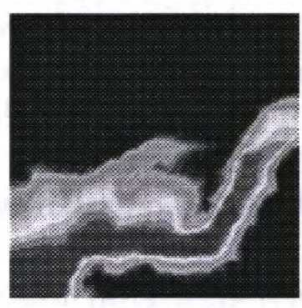

4e: Flow Based Dual CVD Scheme 3.0

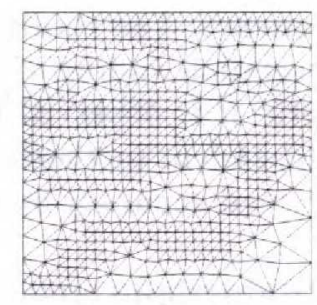

4f: Flow Based Primal Grid
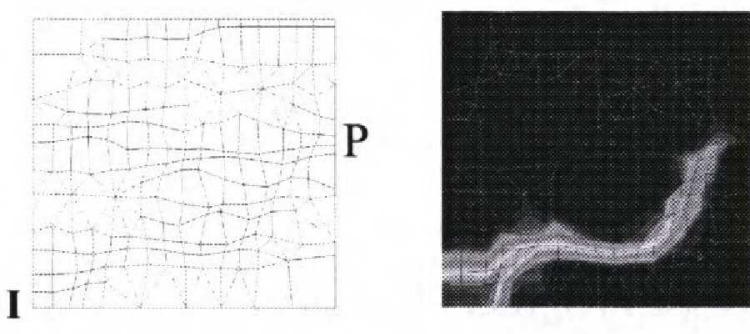

5a: Flow Based Hybrid Dual- Standard CVD Scheme

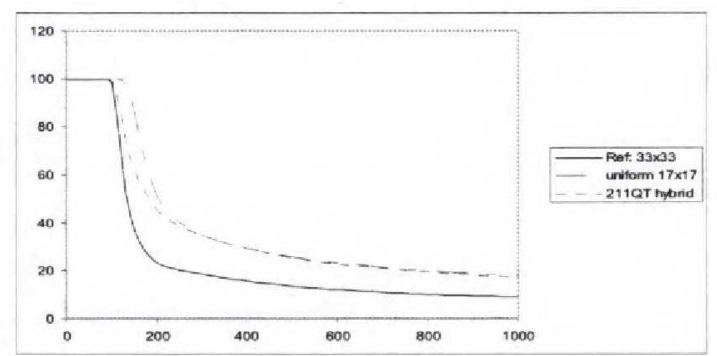

5b: Fractional Flow Comparison 